\title{
Macrophage migration inhibitory factor deficiency enhances immune response to Nippostrongylus brasiliensis
}

\author{
SR Damle ${ }^{1,3}$, RK Martin ${ }^{1,3}$, JV Cross $^{2}$ and DH Conrad ${ }^{1}$
}

Infections with helminth parasites are endemic in the developing world and are a target for intervention with new therapies. Macrophage migration inhibitory factor (MIF) is a cytokine with pleiotropic effects in inflammation and immune responses. We investigated the role of MIF in a naturally cleared model of helminth infection in rodents, Nippostrongylus brasiliensis. At day 7 postinfection, MIF-deficient $\left(\mathrm{MIF}^{-/-}\right)$mice had reduced parasite burden and mounted an enhanced type 2 immune response (Th2), including increased Gata3 expression and interleukin-13 (IL-13) production in the mesenteric lymph nodes (MLNs). Bone marrow reconstitution demonstrated that MIF produced from hematopoietic cells was crucial and Rag1 ${ }^{-1-}$ reconstitution provided direct evidence that $\mathrm{MIF}^{-1-} \mathrm{CD} 4^{+}$T cells were responsible for the augmented parasite clearance. $\mathrm{MIF}^{-1-} \mathrm{CD} 4^{+}$T cells produced less IL-6 postinfection, which correlated with enhanced Th2 responses. $\mathrm{MIF}^{-1-} \mathrm{CD}^{+}$Tcells exhibited lower nuclear factor-кB activation, potentially explaining the reduction in IL-6. Finally, we demonstrated enhanced clearance of the parasite and Th2 response in wild-type mice treated with the MIF tautomerase inhibitor, sulforaphane, a compound found naturally found in cruciferous vegetables. These results are the first to describe the importance of the tautomerase enzyme activity in MIF function in $N$. brasiliensis infection.

\section{INTRODUCTION}

Soil-transmitted helminths infect greater than one billion individuals worldwide, particularly in tropical and subtropical climates where sanitation is poor. These infections include the species Ascaris lumbricoides, Trichuris trichiura, Necator americanus, and Ancylostoma duodenale. ${ }^{1}$ Although these infections are not usually fatal, significant morbidity related to the worm burden in the individual remains a problem. Symptoms range from diarrhea and abdominal pain to general malaise and weakness, but can progress to impaired cognition and physical development, especially in children. ${ }^{1}$ While current antihelminthic agents are available and relatively inexpensive, the global coverage of children with these treatments is only $33 \% .{ }^{2}$ Understanding and enhancing the immune response to soil-transmitted helminths could greatly expand the potential therapeutic options and reduce the helminth-associated morbidity.
Nippostrongylus brasiliensis is a rodent helminth that is similar to the human hookworm. $N$. brasiliensis elicits a dominant type 2 immune response (Th2) response in mice, including the cytokines interleukin-4 (IL-4), IL-5, and IL-13 as well as immunoglobulin E (IgE) antibody production. Downstream effects include enhanced mucus production, goblet cell hyperplasia, eosinophilia, and enteric nerve stimulation, all of which facilitate expulsion of the intestinal worm. This response has been well studied, but in recent years elucidating the effect of the innate immune system on $\mathrm{Th} 2$ responses has increased in importance. ${ }^{3,4}$ Many of these innate factors alter adaptive immune responses toward a specific T-helper subset, thereby altering effective immunity to helminth parasites.

Macrophage migration inhibitory factor (MIF) was one of the first cytokines to be described. ${ }^{5,6}$ Since its initial discovery, it has been shown to have many functions, acting as an anterior pituitary hormone, ${ }^{7}$ a proinflammatory cytokine, ${ }^{8-11}$ and a

\footnotetext{
${ }^{1}$ Department of Microbiology and Immunology, Virginia Commonwealth University, Richmond, Virginia, USA and ${ }^{2}$ Department of Pathology, University of Virginia, Charlottesville, Virginia, USA. Correspondence: DH Conrad (dconrad@vcuhealth.org)

${ }^{3}$ The first two authors contributed equally to this work and are designated as first authors. 
tautomerase enzyme, ${ }^{12-14}$ among many others. ${ }^{15}$ Consistent with its diverse functions and the large array of cell types that produce it, the downstream effects of MIF are extensive: MIF activates mitogen-activated protein kinase signaling pathways, ${ }^{16}$ promotes lipopolysaccharide stimulation through Toll-like receptor $4,{ }^{17}$ interacts with Jab1 to increase transcription of AP-1 target genes, ${ }^{18}$ and activates nuclear factor- $\kappa \mathrm{B}(\mathrm{NF}-\kappa \mathrm{B}) .{ }^{19}$ In addition to these functions and in contrast with other cytokines, MIF encodes an enzymatic activity, acting as a ketoto-enol tautomerase. ${ }^{14}$ The role of this enzymatic activity in the biological functions of MIF remains controversial, as no physiological substrate has been identified. However, several groups have characterized inhibitors of this activity, which display efficacy in disease models that are dependent on MIF. ${ }^{20,21}$

Given that MIF is involved in many cellular pathways and functions as a proinflammatory cytokine, it is not surprising that it is has been studied in both human and murine disease, including rheumatoid arthritis, ${ }^{22}$ atherosclerosis, ${ }^{23}$ and lipopolysaccharide-induced sepsis. ${ }^{8,11,24}$ In infections caused by intracellular pathogens such as Salmonella typhimurium and Mycobacterium tuberculosis, MIF deficiency led to a diminished Th1 immune response and was detrimental to host. ${ }^{25,26}$ MIF has additionally been studied in several parasite infections. ${ }^{27-31}$ However, these studies have been limited to models in which a Th1 response is required for clearance, such as Leishmania major, ${ }^{27}$ Plasmodium chabaudi, ${ }^{30,31}$ and Taenia crassiceps. ${ }^{32}$ To date, inquiry into the role of MIF in infection with parasites requiring a Th2 response for clearance has been limited. ${ }^{29}$

In this study, we analyzed the role of MIF in the immune response of mice infected with $N$. brasiliensis. We examined how MIF deficiency altered the course of infection and the cellular immune responses using $\mathrm{MIF}^{-/}$mice. $\mathrm{MIF}^{-/}$mice had enhanced clearance of $N$. brasiliensis owing to a robust Th2 response. Using an inhibitor of MIF, we demonstrate the importance of the tautomerase enzyme activity in mediating immune alterations and immunity to $N$. brasiliensis.

\section{RESULTS}

\section{MIF deficiency enhances clearance of $\boldsymbol{N}$. brasiliensis}

Given the increasing appreciation of the importance of innate immune system factors in the initiation, amplification, and finetuning of Th2 immune responses, we sought to determine if MIF had a role in the immune response to $N$. brasiliensis. Wild-type (WT) and $\mathrm{MIF}^{-1-} \mathrm{C} 57 \mathrm{Bl} / 6$ mice were infected with $600 \mathrm{~L} 3$ N. brasiliensis larvae. The $\mathrm{MIF}^{-1-}$ mice had fewer eggs per gram (EPG) feces (Figure 1a,b) and fewer adult worms (L5 stage) in the proximal small intestine at day 7 postinfection (Figure 1c). In contrast, there was no difference in the number of L4 stage worms in the lungs at day 2 in $\mathrm{MIF}^{-1-}$ mice (Figure 1d). These data suggest that MIF deficiency primarily affects the immune response to $N$. brasiliensis in the gut. A similar decrease in EPG and adult worms was also seen in $\mathrm{MIF}^{-/-}$mice on the Balb/c background (Supplementary Figure SF1 online). Thus, MIF deficiency leads to enhanced control of $N$. brasiliensis on both $\mathrm{C} 57 \mathrm{Bl} / 6$ and Balb/c backgrounds.

\section{MIF $^{-I-}$ mice develop a heightened Th2 response}

Th2 responses are critical for clearance of $N$. brasiliensis; we examined the spleen and mesenteric lymph nodes (MLNs, the draining lymph node) for markers of this response. MLNs from $\mathrm{MIF}^{-l^{-}}$mice had significantly increased cellularity when compared with WT MLNs (Figure 2a) and in vitro stimulation with anti-CD3e/anti-CD28 resulted in significantly more proliferation in the $\mathrm{MIF}^{-1-}$ cultures, when compared with WT MLN controls (Figure 2b). We measured mRNA expression of transcription factors associated with Th1 and Th2 responses (Tbx21 and Gata3, respectively) in MLNs isolated from mice on day 7 postinfection. $\mathrm{MIF}^{-/-} \mathrm{MLN}$ from infected mice exhibited a 12-fold increase in the expression of Gata3 relative to the uninfected controls, compared with only sevenfold increase in MLNs from WT mice. In contrast, there was no difference in fold induction of Tbx21 (Figure 2c). We also measured the expression of mRNA for the Th2 cytokines, IL-4 and IL-13, which are integral to the clearance of $N$. brasiliensis. There was no difference in the degree of IL-4 induction in the MLNs isolated from WT and $\mathrm{MIF}^{-/-}$mice (Figure 2d). However, IL-13 induction was enhanced in the $\mathrm{MIF}^{-/-}$mice, compared with the WT controls. Expression of Th1 and Th2 transcription factor and cytokines were not altered in uninfected mice (Supplementary Figure SF2C,D). Next, we measured the production of Th1 and Th2 cytokines (interferon$\gamma($ IFN $\gamma)$ and IL-13, respectively) in cultured MLNs from infected mice stimulated with anti-CD3\&/anti-CD28. Supernatants from the cultured MLNs from $\mathrm{MIF}^{-1-}$ mice contained significantly more IL-13 than did the WT MLN supernatant (Figure 2e). In contrast, IFN $\gamma$ was unchanged (Figure 2f). Given that we see increased IL-13, but not IL-4, we examined $\mathrm{MIF}^{-/-}$and WT mice for innate lymphoid cells type 2. We found no differences in the percentage of innate lymphoid cells type 2 in the lungs, pleural lavage, and peritoneal lavage on day 7 after infection (Supplementary Figure SF2). Also, we did not see differences in the percentage of mast cells in the peritoneal lavage (Supplementary Figure SF2). Sections of small intestine stained with Periodic Acid Schiff demonstrate an increase in Periodic Acid Schiff-positive cells in day 7-infected $\mathrm{MIF}^{-1-}$ mice compared with WT (Figure 2g). These results indicate that the $\mathrm{MIF}^{-/-}$mice exhibit a heightened Th2 response in the draining lymph node and in the gut, which may be responsible for enhanced parasite clearance.

\section{MIF deficiency does not impact splenic Th2 response or serum antibody production}

As $\mathrm{MIF}^{-1-}$ mice infected with $N$. brasiliensis exhibited a distinct increase in the Th2 response in the MLN, we investigated the adaptive immune responses in the spleen during infection to determine if the phenomenon observed in the MLN was present in non-draining secondary lymphoid structures. To accomplish this, antibody levels were measured in the serum of $N$. brasiliensis-infected mice at various time points postinfection. We found no difference in either serum IgM- or the Th2-associated antibodies, IgE and IgG1 (Supplementary Figure SF3A-C), as a function of MIF 

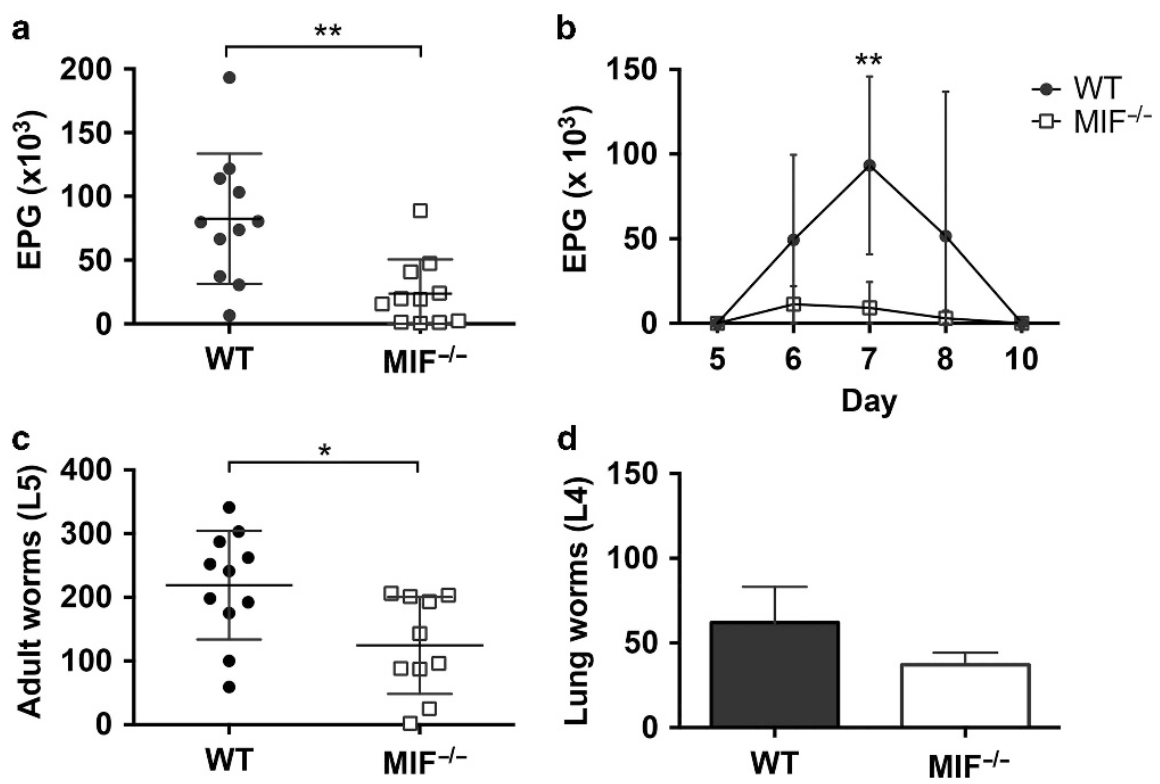

Figure $1 \mathrm{MIF}^{-1-}$ mice exhibit enhanced clearance of Nippostrongylus brasiliensis infection. (a and $\left.\mathbf{b}\right)$ Eggs per gram (EPG) feces were enumerated in wild-type (WT) and $\mathrm{MIF}^{-1-}$ mice at (a) day 7 postinfection and (b) over the course of infection. (c) Number of adult worms (L5) in the proximal small intestine was measured at day 7 postinfection. (d) On day 2 postinfection, the lungs were assessed for lung worms (L4). Symbols represent individual mice and data are combined from at least three independent experiments with three mice per group. ${ }^{* \star} P<0.01$ and ${ }^{*} P<0.05$, unpaired $t$-test. MIF, macrophage migration inhibitory factor.

deficiency. In addition, there were no differences in proliferation and cytokine production in cells isolated from the spleen of $\mathrm{MIF}^{-1-}$ mice compared with WT. Splenocytes isolated from $\mathrm{MIF}^{-1-}$ mice at day 7 after infection proliferated to the same extent as those of WT mice (Supplementary Figure SF4A) and did not produce more IL-13 (Supplementary Figure SF4B). Moreover, the fold induction of Tbx21, Gata3, IL-4, and IL-13 mRNA in the splenocytes following infection was also similar between WT and $\mathrm{MIF}^{-1-}$ mice (data not shown). This indicates that the Th2 response was specifically enhanced in the draining LNs of $\mathrm{MIF}^{-1-}$ mice, and not in the spleen.

\section{Enhanced control of $N$. brasiliensis is dependent on loss of MIF in the hematopoetic compartment}

To determine if MIF deficiency in the hematopoietic compartment was responsible for the phenotype observed in the $\mathrm{MIF}^{-1-}$ mice, we irradiated WT mice and reconstituted them with $\mathrm{MIF}^{-1-}$ bone marrow and compared them with irradiated $\mathrm{MIF}^{-1-}$ mice reconstituted with WT bone marrow. After 6 weeks of reconstitution, mice were infected with $N$. brasiliensis. On day 8 postinfection, WT mice reconstituted with $\mathrm{MIF}^{-1-}$ bone marrow had reduced eggs in the feces compared with $\mathrm{MIF}^{-1-}$ mice reconstituted with WT bone marrow (Figure 3a). We confirmed reconstitution by performing flow cytometry on the blood, spleen, peritoneal lavage cells, and MLN after infection. Successful reconstitution was demonstrated by the presence of CD $45.1^{+}$cells in the $\mathrm{MIF}^{-1-}$ mice reconstituted with WT bone marrow and CD45.2 ${ }^{+}$cells in WT mice reconstituted with $\mathrm{MIF}^{-/-}$bone marrow (Supplementary Figure SF6). This suggests that lack of MIF in hematopoietic-derived cells is important for the enhanced clearance of $N$. brasiliensis.

Next, we examined the tissues in WT mice for relative MIF message levels before and after infection (day 7). Although there was a trend toward increased MIF expression in the spleen $(P=0.07)$, the only significant induction of MIF expression was observed in the proximal small intestine and MLN (Figure 3b). To determine which hematopoietic cell(s) produce MIF in response to $\mathrm{N}$. brasiliensis infection, we sorted $\mathrm{B}, \mathrm{CD} 4^{+} \mathrm{T}$, and dendritic cells from the MLNs. Only CD4 ${ }^{+} \mathrm{T}$ cells $\left(\mathrm{CD} 90.2^{+}\right.$ $\mathrm{CD}^{+}$) exhibited an induction of MIF expression on day 7 postinfection (Figure 3c). Given previous evidence for the role of MIF in macrophages, we also measured MIF expression in macrophages from day 7-infected mice, but we found no increase in expression (Supplementary Figure SF5). Taken together, our data indicate that lack of MIF in the hematopoietic compartment, primarily within $\mathrm{CD} 4^{+} \mathrm{T}$ cells, correlates with an enhanced Th2 immune response and worm expulsion. We sought to prove that $\mathrm{MIF}^{-1-} \mathrm{CD} 4^{+} \mathrm{T}$ cells mediated the effects demonstrated in the $\mathrm{MIF}^{-1-}$ mice. To this end, we reconstituted Rag1 ${ }^{-1-}$ mice, which have no mature $\mathrm{B}$ or $\mathrm{T}$ cells, with WT B cells and either WT or $\mathrm{MIF}^{-1-} \mathrm{CD} 4{ }^{+} \mathrm{T}$ cells. On day 7 after infection with $N$. brasiliensis, non-reconstituted Rag1 ${ }^{-1-}$ mice had a severe infection with the helminth. Rag1 ${ }^{-1-}$ mice reconstituted with $\mathrm{WT} \mathrm{CD} 4^{+} \mathrm{T}$ cells were able to mount a response to $N$. brasiliensis as demonstrated by fewer EPG compared with the non-reconstituted $\mathrm{Rag} 1^{-1-}$. Intriguingly, Rag1 $1^{-1-}$ mice given $\mathrm{MIF}^{-1-} \mathrm{CD} 4^{+} \mathrm{T}$ cells had significantly reduced EPGs compared with the WT control (Figure 4). These data reinforce the importance of $\mathrm{MIF}^{-/-}$ $\mathrm{CD} 4{ }^{+} \mathrm{T}$ cells in the enhanced $\mathrm{Th} 2$ response in $\mathrm{MIF}^{-1-}$ mice. 


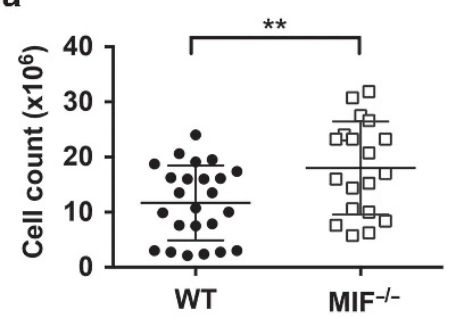

d

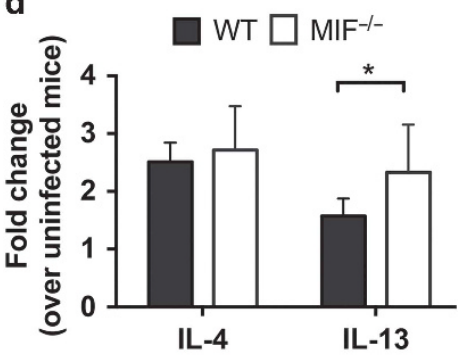

b

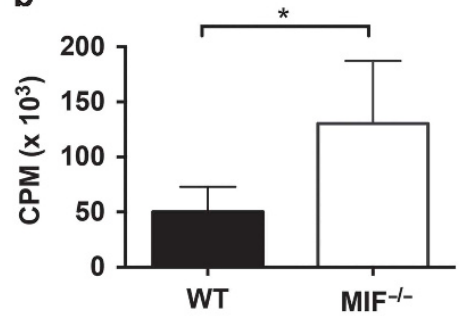

e

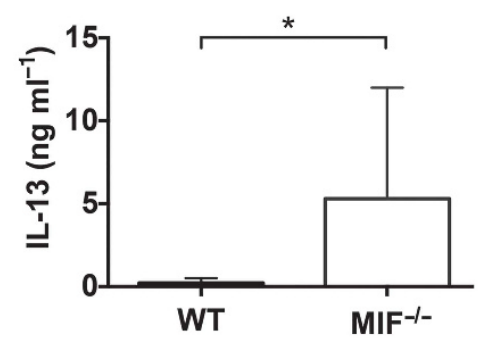

C

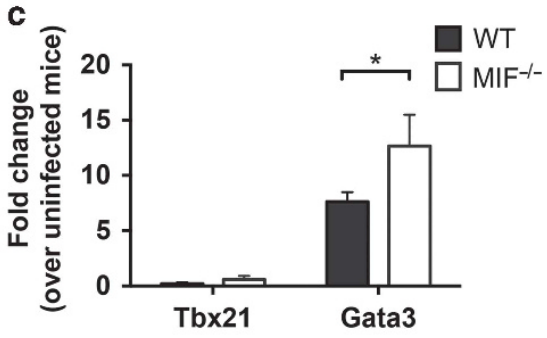

f

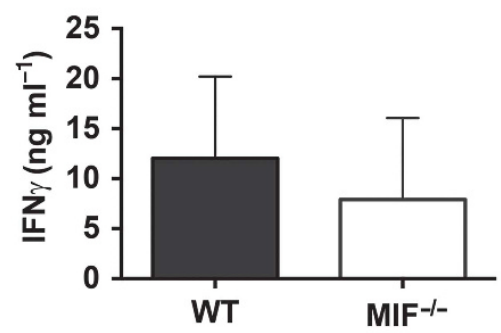

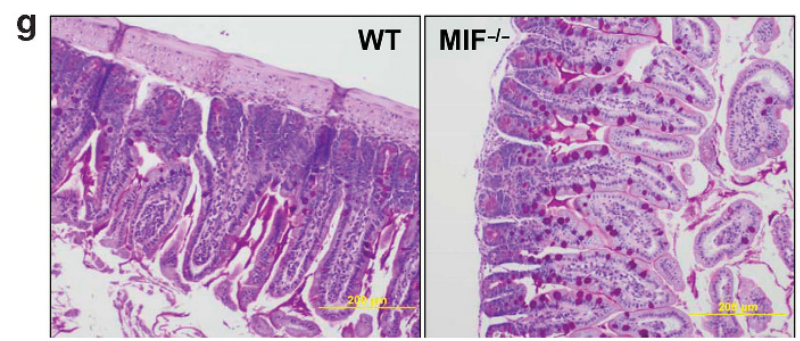

Figure 2 Macrophage migration inhibitory factor (MIF) deficiency enhances the adaptive type 2 immune response (Th2) response to Nippostrongylus brasiliensis. (a) Cells in the mesenteric lymph node (MLN) were enumerated on day 7 postinfection. (b) A total of $1.25 \times 10^{5} \mathrm{MLN}$ cells per ml were seeded in anti-CD3 $\varepsilon$-coated plates with anti-CD28 in the media. Proliferation was assessed by $\left[{ }^{3} \mathrm{H}\right]$ thymidine incorporation (counts per minute $\left.(\mathrm{CPM})\right)$ after $72 \mathrm{~h}$. (c) Fold change in mRNA expression (over uninfected) of transcription factors, Tbx21 and Gata3, was measured in MLN cells on day 7 after infection. (d) Fold change in interleukin-4 (IL-4) and IL-13 mRNA expression was assessed on day 7 postinfection in MLN cells. (e and f) A total of $5 \times 10^{5}$ cells per ml were cultured with anti-CD3\&/anti-CD28 stimulation for $96 \mathrm{~h}$ and (e) IL-13 and (f) interferon- $\gamma$ (IFN $\gamma$ ) were measured in the culture supernatant by enzymelinked immunosorbent assay (ELISA). (g) Sections of the small intestine from day 7-infected wild-type (WT) and MIF ${ }^{-1-}$ mice were stained with Periodic Acid Schiff. Symbols represent individual mice and data are combined from at least three independent experiments each with three mice per group. ${ }^{\star *} P<0.01$ and ${ }^{\star} P<0.05$, unpaired $t$-test.

\section{MIF $^{-I-}$ CD4 $^{+}$T cells exhibit reduced NF-кB signaling and IL-6 production}

Recent literature has demonstrated a regulatory role for IL-6 in Th2 immune responses to the intestinal helminth Heligmosomoides polygyrus in that $\mathrm{CD} 4{ }^{+} \mathrm{T}$ cells from IL-6-deficient mice produced more Th 2 cytokines. ${ }^{33}$ As $\mathrm{CD} 4{ }^{+}$T cells had the largest increase in MIF message in response to $N$. brasiliensis infection, we investigated the induction of IL- 6 in these cells. $\mathrm{CD}^{+}{ }^{+} \mathrm{T}$ cells isolated from the MLN of from $\mathrm{MIF}^{-/-}$mice had a modest increase (fivefold) in IL-6 message expression at day 7 postinfection with $N$. brasiliensis. In contrast, IL-6 message was increased 38 -fold in the MLN of WT mice (Figure 5a). Additionally, IL-17a expression was reduced in the $\mathrm{MIF}^{-/-} \mathrm{CD}^{+}{ }^{+} \mathrm{T}$ cells (Figure $5 \mathbf{b}$ ), which is consistent with in the impaired IL-17 expression observed in IL-6-deficient mice after helminth infection. ${ }^{33}$

To understand how MIF deficiency could alter IL-6 production, we examined the pathways on which MIF is known to act. MIF activates mitogen-activated protein kinase pathways. ${ }^{16}$ To determine if this pathway was involved in the reduction of IL-6 expression, phosphorylation of ERK1/2 in $\mathrm{MIF}^{-/-} \mathrm{MLN} \mathrm{CD}^{+} \mathrm{T}$ cells on day 7 after infection was measured by western blot. No difference in ERK1/2 phosphorylation between WT and $\mathrm{MIF}^{-/-}$was observed (Figure 5c,d). MIF is also known to act through canonical NF- $\kappa B$ pathways by increasing signaling through Toll-like receptor 4 and other yetto-be determined mechanisms leading to the expression of target genes. ${ }^{19}$ On day 7 after infection, significantly less phosphorylation of NF- $\mathrm{KB}$ component, p65, was observed in $\mathrm{MIF}^{-/-} \mathrm{CD}^{+}{ }^{+} \mathrm{T}$ cells (Figure 5e,f). As IL-6 is a known NF$\kappa \mathrm{B}$ target gene, ${ }^{34}$ these results suggest that the reduced induction of IL- 6 in the MIF-deficient CD4 ${ }^{+} \mathrm{T}$ cells may be a consequence of impaired NF- $\mathrm{KB}$ activation.

\section{Administration of MIF tautomerase inhibitor, SFN, enhances clearance of $\boldsymbol{N}$. brasiliensis}

The cytokine MIF has been characterized as a keto-enol tautomerase. $^{12,14}$ Although the precise endogenous substrates for this enzymatic activity are unknown, MIF tautomerizes the artificial substrates D-dopachrome and 


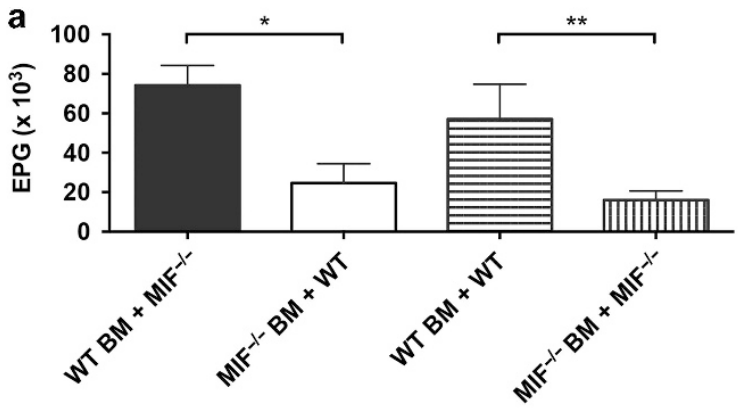

c

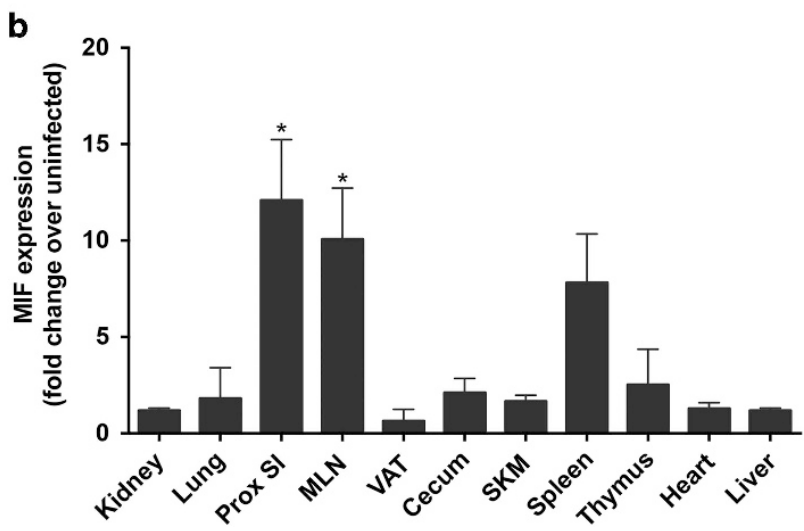

Figure 3 Macrophage migration inhibitory factor (MIF) deficiency in the hematopoietic compartment is sufficient for phenotype. (a) MIF $^{-/-}$mice $^{-1}$ reconstituted with WT bone marrow (WT BM $+\mathrm{MIF}^{-1-}$ ) and WT mice reconstituted with $\mathrm{MIF}^{-1}$ - bone marrow (MIF ${ }^{-1-} \mathrm{BM}+\mathrm{WT}^{-}$) were infected with Nippostrongylus brasiliensis and eggs per gram (EPG) was measured at day 8. (b) Tissues from day 7-infected and -uninfected WT mice were flash frozen and total RNA was isolated. MIF expression was measured as fold change of infected over uninfected mice. (c) B cells (B220 ${ }^{+}$CD90.2 ${ }^{-}$), dendritic cells $\left(\mathrm{CD} 90.2^{-} \mathrm{CD} 11 \mathrm{c}^{+}\right)$, and $\mathrm{CD} 4^{+} \mathrm{T}$ cells $\left(\mathrm{B} 220^{-} \mathrm{CD} 90.2^{+} \mathrm{CD} 4^{+}\right)$were sorted from day 7 -infected and -uninfected mesenteric lymph nodes (MLNs). MIF expression was measured and fold change was calculated. Data are combined from two independent experiments of three mice per group in each experiment. (a and $\mathbf{c}$ ) ${ }^{\star} P<0.05$, unpaired $t$-test and (b) one-way analysis of variance (ANOVA).

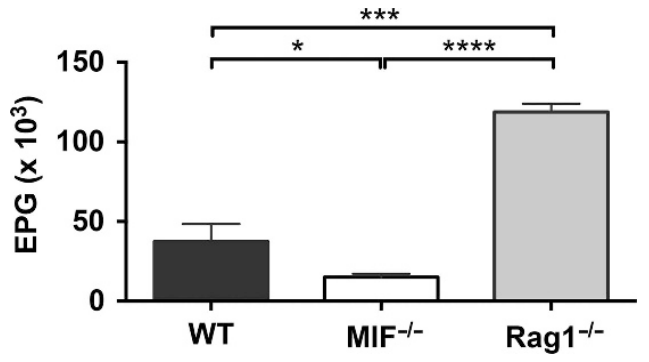

Figure $4 \mathrm{MIF}^{-1-} \mathrm{CD}^{+} \mathrm{T}$ cells mediate enhanced clearance of Nippostrongylus brasiliensis. (a) Rag1 ${ }^{-1-}$ mice, reconstituted with (b) $20 \times 10^{6} \mathrm{WT} \mathrm{B}^{2} 20^{+}$cells and $10 \times 10^{6} \mathrm{WT}$ or MIF $^{-l-} \mathrm{CD} 4^{+}$T cells, were injected with $N$. brasiliensis. Eggs per gram (EPG) feces were measured on day 7 postinfection. Representative data are shown from one of two experiments, $n=3$ mice per group per experiment. ${ }^{*} P<0.05,{ }^{* * \star} P<0.001$ and ${ }^{* * * *} P<0.0001$, one-way analysis of variance (ANOVA) with Tukey's multiple comparison test. MIF, macrophage migration inhibitory factor.

phenylpyruvate, providing the means to assay its activity in vitro. $R, S$-sulforaphane ((1-isocyanato- $4 R$ (methylsulfinyl)butane), SFN) is a naturally occurring organosulfur compound found in cruciferous vegetables such as broccoli and brussel sprouts. ${ }^{35}$ SFN covalently modifies the N-terminal proline of MIF, which functions as the catalytic center for its tautomerase activity. ${ }^{20,36,37}$ Therefore, SFN is a potent and irreversible inhibitor of MIF.

To determine whether the tautomerase activity of MIF is important for its function during N. brasiliensis infection, we administered the tautomerase inhibitor SFN to WT mice daily beginning on the day of infection. Intriguingly, WT mice treated with SFN had fewer eggs in the feces on day 7 of infection relative to the saline controls (Figure 6a) and indeed looked very similar to the $\mathrm{MIF}^{-1-}$ animals (Figure 1a). The SFN-treated group also had fewer adult worms (L5) in the proximal small intestine on day 7 postinfection (Figure $6 \mathbf{b}$ ). To exclude any effects that SFN may have on the enhanced worm clearance in the $\mathrm{MIF}^{-1-}$ mice, we examined SFN treatment in $\mathrm{MIF}^{-1-}$ mice and observed no effect of SFN on the clearance of $N$. brasiliensis in the absence of MIF (Figure 6c). To exclude effects of SFN functioning through its ability to induce antioxidant response element-dependent gene expression, we measured the expression of genes regulated by this transcription factor. We found no significant difference in the expression of these genes in the MLNs of naïve and $N$. brasiliensis-infected WT mice with or without SFN treatment (Supplementary Figure SF7), although there was a slight trend upwards $(P=0.06)$ for NADPH quinone oxidoreductase (Nqo1). We also measured the levels of Th2 cytokines in day 7-infected saline and SFN-treated mice. In agreement with the EPG data, SFN-treated mice had a significant increase in the expression of IL- 4 and IL-13 in the MLNs compared with saline treatment (Figure 6d,e). This suggests that the tautomerase activity of MIF is essential for its normal function in the immune response to $N$. brasiliensis. 


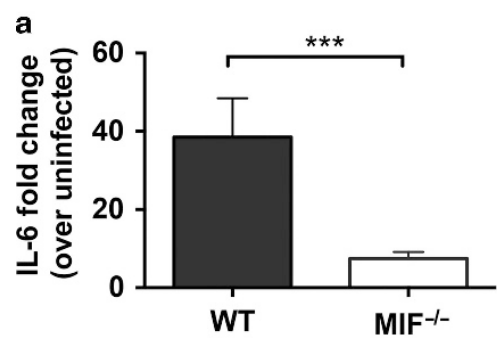

C

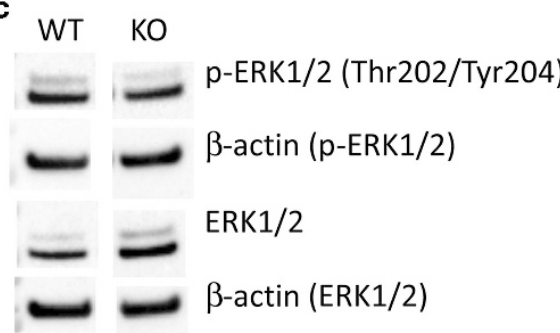

e

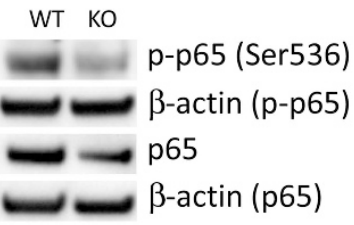

b

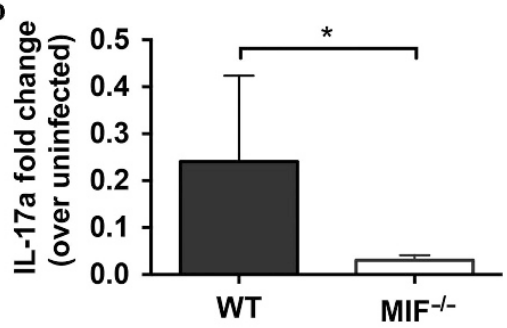

d
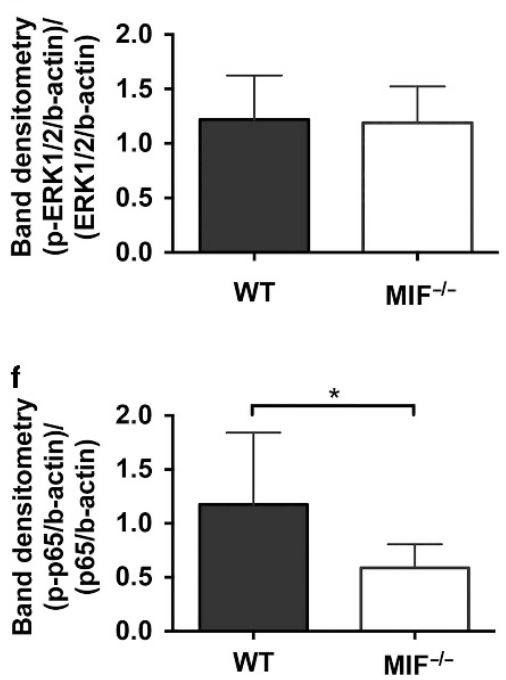

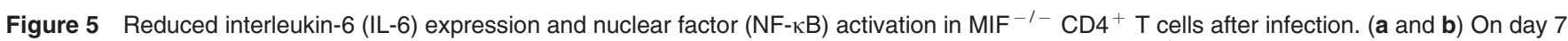

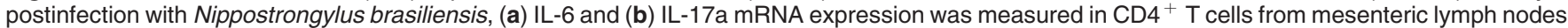
(MLNs). Data are expressed as fold change over uninfected mice. (c-f) CD4 ${ }^{+}$T cells isolated from the MLNs of day 7 -infected mice were lysed and immunoblots performed for the indicated proteins. (c and e) Representative immunoblots. (d and f) Quantification of (d) phosphorylated ( $p$ )-ERK $1 / 2$ and ERK1/2 and (f) p-p65 and p65. For each, the band densities were normalized to their respective $\beta$-actin controls. The ratios of normalized p-ERK1/2 and p-p65 to normalized total ERK1/2 and p65, respectively, were calculated. Data were combined from (c and d) one experiment of three mice per group and from (a, b, e, and f) three independent experiments each with three mice per group. ${ }^{* \star} P<0.001$ and ${ }^{\star} P<0.05$, unpaired $t$-test. ERK, extracellular signalregulated kinase; $\mathrm{KO}$, knockout; MIF, macrophage migration inhibitory factor; WT, wild type.

\section{DISCUSSION}

MIF has been studied in the context of both the innate and adaptive immune responses in many disease models. ${ }^{38}$ The $\mathrm{T}$ cell was the first identified source of $\mathrm{MIF}^{5,6}$ and has been extensively studied. Here, we describe the role of MIF and, more specifically, its enzymatic tautomerase activity, in the immune response to $N$. brasiliensis. We have demonstrated that MIF deficiency results in decreased induction of IL-6 following infection, correlating with an enhanced Th2 response and improved control of the infection in $\mathrm{MIF}^{-/-}$mice.

During infection with $N$. brasiliensis, cells isolated from the MLNs of $\mathrm{MIF}^{-/-}$mice exhibited increased T-cell proliferation in response to TCR stimulation and produced more IL-13 with no reduction in IFN $\gamma$ production. Although IL-4 induction was not significantly changed, IL-13 alone is sufficient to enhance the 'weep and sweep' response that leads to worm expulsion. ${ }^{39}$ This was accompanied by increased expression of the Th2 transcription factor Gata3. In a different helminth infection model, T. crassiceps, ${ }^{29}$ which also elicits a Th2-like immune response, ${ }^{40} \mathrm{MIF}^{-1-}$ mice were shown to be more susceptible to the parasite and had a larger parasite burden despite enhanced IL-13 production. In cysticercosis, it is thought that nitric oxide (NO) from macrophages provides the bulk of protection. Thus, in spite of enhanced IL-13 production, it is possible that $\mathrm{MIF}^{-1-}$ mice were susceptible to $T$. crassiceps because $\mathrm{MIF}^{-1-}$ macrophages are unable to produce the high levels of TNF and NO necessary for parasite clearance ${ }^{29}$ in this model. Similarly, in Schistosoma japonicum infection, mice given a neutralizing anti-MIF IgG exhibited increased worm burden in the liver. This phenotype was also attributed to the lack of NO and TNF produced by macrophages. ${ }^{41}$ This suggests that the impact of MIF on the immune response to a given parasite will vary as a function of the dominant mechanism(s) of worm clearance.

Although many cell types express MIF, we found that MIF deficiency in the hematopoietic compartment was sufficient to recapitulate the enhanced immunity to $N$. brasiliensis that we found in $\mathrm{MIF}^{-1-}$ mice. Examining the expression of MIF in many different tissues and cell types indicated that $\mathrm{T}$ cells are likely the major source of MIF induced by infection with $N$. brasiliensis. Our Rag $1^{-1-}$ reconstitution data (Figure 4) further support this conclusion. These data provide direct evidence that $\mathrm{MIF}^{-/-} \mathrm{CD}^{+} \mathrm{T}$ cells confer an advantage in the immune response to $N$. brasiliensis compared with WT. 
a

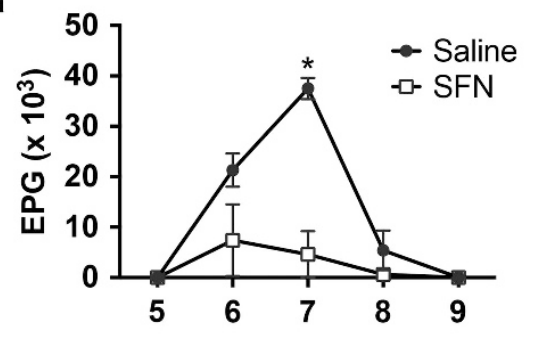

c

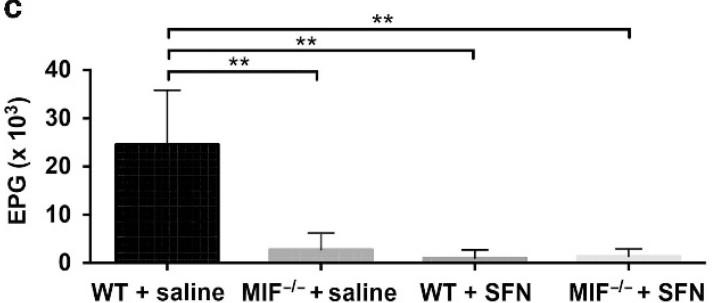

b

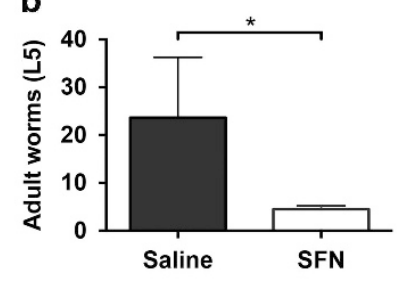

d

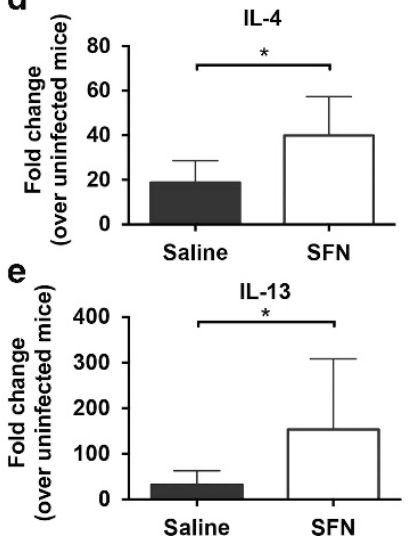

Figure 6 Macrophage migration inhibitory factor (MIF) inhibitor and natural product, sulforaphane (SFN), promotes clearance of Nippostrongylus brasiliensis in wild-type (WT) mice. (a and b) WT mice were infected with N. brasiliensis. Saline or $200 \mu \mathrm{g}$ SFN was administered intraperitoneally into WT mice daily beginning on the day of infection. Eggs per gram (EPG) feces were enumerated on indicated days (a). On day 7 of infection, adult worms were measured in the proximal small intestine (b). (c) WT and $\mathrm{MIF}^{-}{ }_{-}^{-}$mice were infected with $N$. brasiliensis and given either saline or $200 \mu \mathrm{g}$ SFN daily beginning on the day of infection. EPG was measured on day 7 postinfection. (d and e) Fold change of interleukin-4 (IL-4) and IL-13 were measured in the mesenteric lymph nodes (MLNs) of day 7 infected saline and SFN-treated WT mice. Data are representative of one experiment of three performed each with three mice per group. (a, $\mathbf{b}, \mathbf{d}$, and $\mathbf{e})^{* \star} P<0.01$ and ${ }^{*} P<0.05$, unpaired $t$-test and (c) one-way analysis of variance (ANOVA) with a Dunnett's multiple comparison test to examine differences from WT + saline group.

To further investigate the mechanism through which MIF influences worm clearance, we examined the impact of MIF deficiency on infection-induced IL-6. Recently, IL-6 was shown to be detrimental to the Th2 response and immunity to the intestinal helminth, H. polygyrus. ${ }^{33}$ Mice deficient in IL-6 had reduced parasite burden and enhanced $\mathrm{Th} 2$ response. Without IL-6 during this infection, the T-helper response was shifted away from Th17 and toward Th2 and regulatory T cells but with less Foxp3, Helios, and Gata3 transcription factor expression. MIF has directly or indirectly been shown to influence the production of many molecules important in inflammation, including TNF, IFN $\gamma$, IL-1B, IL-2, IL-6, IL-8, MIP2 (macrophage inflammatory protein 2), ${ }^{8-11} \mathrm{NO}^{7,24} \mathrm{PGE}_{2}$ (prostaglandin E2), and $\mathrm{COX}_{2}$ (cyclooxygenase-2). ${ }^{16}$ MIF-deficient macrophages are the most well studied and have been demonstrated to produce fewer inflammatory cytokines upon stimulation or infection. ${ }^{38}$ In this study, MIF deficiency resulted in less IL-6 induction in the $\mathrm{MIF}^{-1-} \mathrm{CD} 4 \mathrm{~T}$ cells (Figure 5a) as well as a decrease in IL-17a expression following infection (Figure 5b). In the H. polygyrus model, it was posited that, without IL-6, the response shifted away from Th17 and towards Th2 and an altered Treg phenotype. ${ }^{33}$ Our results largely support these conclusions, although we did not see a corresponding increase in IL-10 expression (data not shown). This is likely due to the fact that IL-6 is reduced but not absent in our model as compared with the aforementioned IL-6-deficient mice. It may also possibly reflect differences between
$N$. brasiliensis and $H$. polygyrus infection models, namely the former is efficiently controlled by the Th2 response whereas the latter establishes a more chronic infection.

The tautomerase activity of MIF was first described by Rosengren et al. ${ }^{12}$ Several isothiocyanate compounds have been characterized as irreversible inhibitors of this enzymatic activity, ${ }^{20,36,37}$ including SFN. Although the role of MIF's enzymatic activity in its biological functions remains controversial, several published reports have supported the contention that the tautomerase activity is essential for many functions of MIF. ${ }^{42}$ Particularly interesting is the study by Simpson et al. $^{42}$ in which MIF-deficient tumors exhibited reduced growth and impaired ability to attract myeloid derived suppressor cells. They were able to demonstrate that the MIF tautomerase enzyme was critical for this effect using both an enzymatically inactive point mutant of MIF and administration of SFN to the mice. ${ }^{42}$ Although many MIF inhibitors are commercially available, we chose to use the compound SFN because of its status as a natural product with a high concentration in cruciferous vegetables. ${ }^{35} \mathrm{SFN}$ is also commercially available in nutritional supplements, making it an even more attractive potential solution to reducing worm burden in patients. It is important to note that, in addition to inhibiting MIF enzyme activity, SFN also induces genes associated with cytoprotective responses including the genes regulated by Keap1-Nrf2-ARE signaling system. ${ }^{43}$ This system protects against damage by oxidant, electrophile, and 
inflammatory stresses. ${ }^{44}$ However, we did not observe induction of antioxidant response element-dependent genes in our SFN-treated mice. In addition, we observed no additional impact of SFN on parasite clearance in $\mathrm{MIF}^{-/-}$mice, suggesting that MIF inhibition is the predominant target in our model. SFN treatment enhanced Th2 transcription factor and cytokine expression in MLNs after $N$. brasiliensis infection, mirroring the response seen in MIF deficiency. These results are the first to show that blockade of the enzyme activity of MIF is sufficient for the enhanced clearance of this helminth infection.

Taken together, our results demonstrate that MIF deficiency leads to an enhanced Th2 immune response against the helminth parasite, $N$. brasiliensis. Although this is not the first example of MIF deficiency resulting in an enhanced Th2 response, this study shows that, in a pathogen model in which the Th2 response is able to control the infection, MIF deficiency or inhibition will further enhance this clearance. This enhancement is due to $\mathrm{CD} 4{ }^{+} \mathrm{T}$ cells, which have the most heightened MIF expression postinfection and in our Rag $1^{-/-}$reconstitution model-mediated parasite clearance. The data further show that $\mathrm{CD}^{+} \mathrm{T}$ cells from $\mathrm{MIF}^{-/-}$mice have reduced IL-6 message, potentially due to impaired $N F-\kappa B$ activation. In conjunction with other reports, this suggests a mechanism for the increased Th2 response, as IL- 6 is known to impede the Th2 response. ${ }^{33}$ Perhaps most excitingly, we were able to show that by blocking the tautomerase enzyme activity of MIF with the inhibitor SFN, we could mimic the response seen in MIFdeficient mice with $N$. brasiliensis infection, suggesting that this nutritional supplement would enhance resistance to parasitic pathogens in which a Th2 response is responsible for control.

\section{METHODS}

Mice. All animal experiments were performed with the approval of the Virginia Commonwealth University Institutional Animal Care and Use Committee. Mice were maintained in the Virginia Commonwealth University animal facility in accordance with guidelines for the humane treatment of laboratory animals set forth by the National Institutes of Health and the American Association for the Accreditation of Laboratory Animal Care. $\mathrm{MIF}^{-1-}$ Balb/c mice were obtained from cryopreserved stocks at the Jackson Laboratory (Bar Harbor, ME). ${ }^{24}$ These mice were then backcrossed onto C57Bl/6 as described. ${ }^{45}$ Female and male WT C57Bl/6 (stock no. 000664) and Balb/c mice (stock no. 000651) aged 6-12 weeks old were purchased from the Jackson Laboratory and used as controls for $\mathrm{MIF}^{-7-} \mathrm{C} 57 \mathrm{Bl} /$ 6 and Balb/c mice, respectively. The C57Bl/6 congenic strain CD45.1 (B6.SJL-Ptprc ${ }^{a} P e p c^{b} /$ BoyJ; stock no. 002014) was used as WT recipient and donor in the bone marrow reconstitution experiment. Mice were killed by isoflurane inhalation followed by cervical dislocation as per AALAC guidelines.

$\boldsymbol{N}$. brasiliensis infection. $N$. brasiliensis cultures were maintained in the lab and infection was performed as described previously. ${ }^{46}$ Briefly, 600 third-stage (L3) N. brasiliensis larvae were injected subcutaneously into mice. Eggs in the feces were enumerated using a saturated $\mathrm{NaCl}$ solution and a McMaster counting chamber. Adult worms (L5) were isolated from the proximal small intestine and counted using a dissecting microscope. ${ }^{46}$

MLN and splenocyte culture and proliferation measurement. Spleens were crushed manually between two frosted microscope slides, filtered through $40 \mu \mathrm{m}$ cell strainer, and red blood cells were lysed using
ACK Lysis Buffer (Quality Biological, Gaithersburg, MD). Splenocytes were washed in phosphate-buffered saline and enumerated. MLNs were isolated, teased apart with forceps, filtered through a $40 \mu \mathrm{M}$ cell strainer, and enumerated. The 96-well plates were coated with antiCD3E $\left(1 \mu \mathrm{g} \mathrm{ml}^{-1}\right.$, clone 145-2C11; Biolegend, San Diego, CA) for $1 \mathrm{~h}$ at $37^{\circ} \mathrm{C}$ and then washed with phosphate-buffered saline. Splenocytes and MLN cells were seeded at $5 \times 10^{5}$ cells per $\mathrm{ml}$ in complete RPMI, RPMI-1640 containing 10\% heat-inactivated fetal bovine serum (Atlanta Biological, Norcross, GA), $2 \mathrm{M} \mathrm{L}$-glutamine, $50 \mu \mathrm{g} \mathrm{ml}^{-1}$ penicillin, $50 \mu \mathrm{g} \mathrm{ml}^{-1}$ streptomycin, $1 \mathrm{~mm}$ sodium pyruvate, $50 \mu \mathrm{M} 2$ mercaptoethanol, $1 \times$ non-essential amino acids, $20 \mathrm{~mm}$ HEPES buffer (all from Invitrogen, San Diego, CA), and with anti-CD28 $\left(2 \mu \mathrm{g} \mathrm{ml}^{-1}\right.$, clone 37.51 ; Biolegend). Cell culture supernatants were harvested after $96 \mathrm{~h}$ of growth at $37^{\circ} \mathrm{C}$ with $5 \% \mathrm{CO}_{2}$. For proliferation assessment, $1.25 \times 10^{5}$ cells ml $^{-1}$ were plated in complete RPMI with anti-CD28 $\left(2 \mu \mathrm{g} \mathrm{ml}^{-1}\right)$. After $72 \mathrm{~h}$ of growth, $1 \mu \mathrm{C}$ of $\left[{ }^{3} \mathrm{H}\right]$ thymidine (Perkin-Elmer, Waltham, MA) was added to each well. After $18 \mathrm{~h}$, cells were harvested onto GFC plates using a Filtermate cell harvester and incorporation of $\left[{ }^{3} \mathrm{H}\right]$ thymidine was measured by beta counter (TopCount plate counter; Perkin-Elmer).

Enyzme-linked immuosorbent assay. Serum was collected at different time points after infection. The following in-house enzymelinked immunosorbent assay (ELISA) antibodies were used in IgE, IgG1, and IgM ELISAs: ${ }^{47}$ anti-IgE capture, biotinylated anti-mouse IgE, anti-mouse IgG1 capture, anti-mouse IgG1 alkaline phosphatase, anti-mouse IgM capture, and anti-mouse IgM alkaline phosphatase. The 96-well plate was coated with the capture antibody; serially diluted samples were added and followed by detection antibody. IgE, IgG1, and IgM standards were used to generate a standard curve. Plates were developed by adding alkaline phosphate substrate (Sigma-Aldrich, St. Louis, MO) and read by a spectrophotometer at 405 and $650 \mathrm{~nm}$.

Cell culture supernatants were examined for IL-13 and IFN cytokines. For IL-13, 96-well plates were coated with anti-mouse IL-13 capture antibody $\left(6 \mu \mathrm{g} \mathrm{ml}^{-1}\right.$, no. MAF413; R\&D Systems, Minneapolis, MN) for $1 \mathrm{~h}$ at $37^{\circ} \mathrm{C}$. Plates were blocked for $1 \mathrm{~h}$ at $37^{\circ} \mathrm{C}$ with $10 \%$ fetal bovine serum in phosphate-buffered saline. Samples were added to plates neat and diluted $1: 10$ for $2 \mathrm{~h}$ at $37^{\circ} \mathrm{C}$. Recombinant IL-13 was used to generate a standard curve. Biotinylated anti-mouse IL-13-detected antibody $\left(0.2 \mu \mathrm{g} \mathrm{ml}^{-1}\right.$, no. BAF413, R\&D Systems) was added for $1 \mathrm{~h}$ at $37^{\circ} \mathrm{C}$, followed by streptavidin-alkaline phosphatase (Southern Biotech, Birmingham, AL) for $1 \mathrm{~h}$ at $37^{\circ} \mathrm{C}$. Plate was developed as described above. IFN $\gamma$ was detected in the supernatants using Ready-Set-Go! IFN $\gamma$ ELISA Kit (eBioscience, San Diego, CA) per the manufacturer's instructions.

Quantitative PCR. Tissues were flash frozen in liquid $\mathrm{N}_{2}$ and total RNA was extracted using TRIzol reagent (Life Technologies, Grand Island, NY). Cells were homogenized in the TRIzol reagent and total RNA was isolated as per the manufacturer's instructions. RNA was quantified using the absorbance at $260 \mathrm{~nm}$ (ND-100 Nanodrop). Four hundred nanograms of RNA was reverse transcribed into cDNA using iScript cDNA Synthesis Kit (Bio-Rad, Hercules, CA). Real-time PCR was performed using the iQ5 system (Bio-Rad). Gene Expression Master Mix and Taqman probes (IL-13 (Mm00434204_m1), IL-4 (Mm00445259_m1), IL-6 (Mm00446190_m1), IL-17a (Mm00439618_m1) and Gapdh (Mm99999915_g1)) were purchased from Applied Biosystems (Grand Island, NY) and used as per the manufacturer's instructions. Primers for Gata3, Tbx21, and $\beta 2$-microglobulin (Supplementary Figure SF8) were purchased from Integrated DNA Technologies (Coralville, IA) and used with SYBR Green SuperMix (Bio-Rad). Fold change from uninfected samples was calculated using the $\Delta \Delta \mathrm{Ct}$ method. ${ }^{48}$

Histology. Small intestines were fixed in $10 \%$ formaldehyde. Paraffin sections were cut, mounted on slides, and stained with Periodic Acid Schiff stain (Life Technologies, Carlsbad, CA). Images were taken on an Olympus BX41 microscope (Olympus, Melville, NY). 
Bone marrow reconstitution. Bone marrow cells were collected from WT CD45.1 and $\mathrm{MIF}^{-1-}$ mice as follows: tibiae and femurs were isolated from the mice and then centrifuged in microcentrifuge tubes to retrieve bone marrow, red blood cells were lysed with ACK Lysing Buffer and then bone marrow cells were washed in phosphate-buffered saline and enumerated. WT CD45.1 and $\mathrm{MIF}^{-1-}$ mice were irradiated (MDS Nordion cell 40 research irradiator $\left[{ }^{137} \mathrm{Cs}\right]$ ) with two doses of $550 \mathrm{cGy}$ separated by a $2 \mathrm{~h}$ rest period. A total of $5 \times 10^{6}$ bone marrow cells were then injected intravenously into the irradiated mice. ${ }^{49}$ After 6-8 weeks of reconstitution, mice were infected with $N$. brasiliensis as described above. Successful reconstitution was indicated by flow cytometric analysis of the spleen, MLN, resident peritoneal cells isolated by lavage, and blood of recipient mice (Supplementary Figure SF4).

Rag1 $^{-1-}$ reconstitution. Rag1 ${ }^{-1-}$ mice (stock no. 002216) were obtained from Jackson Laboratory (Bar Harbor, ME). B cells were isolated from WT spleens using $\mathrm{B} 220^{+}$magnetic bead selection and WT and $\mathrm{MIF}^{-1-}$ splenic CD4 ${ }^{+}$T cells were isolated using L3T4 magnetic bead selection according to the manufacturer's instructions (Miltenyi Biotec, Gergisch, Gladbach, Germany). A total of $20 \times 10^{6} \mathrm{~B}$ cells and $10 \times 10^{6} \mathrm{WT}$ or $\mathrm{MIF}^{-1-} \mathrm{CD} 4^{+} \mathrm{T}$ cells were intravenously injected into Rag1 ${ }^{-1-}$ mice. After 1 week, $N$. brasiliensis was injected as described above.

Flow cytometric analyses and FACS. Single-cell suspensions were obtained from MLNs, spleens, peritoneal lavage, and peripheral blood after bone marrow reconstitution and $N$. brasiliensis infection. Cells were blocked with unlabeled anti-mouse CD16/32 (clone 2.4G2) for $10 \mathrm{~min}$ on ice, followed by incubation with fluorochrome-labeled antibodies. Antibodies used were as follows: Alexa647 anti-mouse CD45.1 (clone A20), PerCP-Cy5.5 anti-mouse CD45.2 (clone 104) (Biolegend), FITC lineage cocktail, CD127 (clone A7R34), ST2 (clone DIH9), CD117 (clone 2B8), and Fcer1 $\alpha$ (clone MAR-1) (Biolegend). Cells were analyzed on a BD Canto Flow analyzer (BD Biosciences, San Jose, CA); data were analyzed using the FlowJo software (version7.6; TreeStar, Ashland, OR). For FACS, single-cell suspensions of MLNs were stained with PE anti-mouse CD90.2 (clone 30.H12), APC anti-mouse/human B220 (clone RA3-6B2), PE-Cy7 anti-mouse CD4 (clone GK1.5), and FITC anti-mouse CD11c (clone N418) (Biolegend) and then sorted on a BD FACS Aria cell sorter (BD Biosciences, San Jose, CA).

MLN CD4 ${ }^{+}$T-cell isolation and western blotting. Single-cell suspensions of MLNs were generated as described above. CD4 ${ }^{+}$cells were isolated by positive selection using non-activating $\mathrm{CD}^{+}{ }^{+}$(clone L3T4) magnetic beads (Miltenyi Biotec) as per the manufacturer's instructions. Total cell lysates from MLN CD4 ${ }^{+} \mathrm{T}$ cells on day 7 postinfection were obtained using lysis buffer (no. 9803; Cell Signaling, Danvers, MA) as per the manufacturer's instruction. Protein was quantified using a Bradford assay (Bio-Rad), and read at $595 \mathrm{~nm}$ on a spectrophotometer. Thirty micrograms of protein was loaded onto Novex NuPage $10 \%$ Bis-Tris gel, run for $30 \mathrm{~min}$ at $70 \mathrm{~V}$ and $1.5 \mathrm{~h}$ at $100 \mathrm{~V}$, and then transferred to a nitrocellulose membrane. Blots were probed with rabbit anti-phospho ERK1/2 (no. 4377; Cell Signaling), rabbit anti-ERK1/2 (no. 9102; Cell Signaling), rabbit anti-phospho p65 (no. 3033; Cell Signaling), rabbit anti-p65 (no. 8242; Cell Signaling), and horse radish peroxidase anti- $\beta$-actin (Sigma-Aldrich). Signal was detected using Clarity Western ECL Blotting Substrate (Bio-Rad) and a ChemiDoc MP System (Bio-Rad) was used to measure band densitometry.

Sulforaphane administration. Indicated mice were given daily intraperitoneal injections of $200 \mu \mathrm{g}$ SFN (LKT Labs, St Paul, MN) in saline at the beginning on the day of infection. ${ }^{42}$

Statistics. All statistical analyses were performed using Prism6 (GraphPad Software, La Jolla, CA). Statistical significance was assessed by two-tailed, unpaired Student's $t$ test (two groups) or one-way analysis of variance for multiple groups with a Tukey's post hoc test or Dunnett's multiple comparisons test. Unless otherwise indicated, differences are not significant. ${ }^{* * *} P<0.0001$, ${ }^{* *} P<0.001$, ${ }^{* *} P<0.01$, and ${ }^{\star} P<0.05$.

SUPPLEMENTARY MATERIAL is linked to the online version of the paper at http://www.nature.com/mi

\section{ACKNOWLEDGMENTS}

We thank Sana Vohra for technical assistance. This study was funded, in part by, NIH/NCl 1F30CA192836 (to SRD) and NIH/NIAID RO1Al18697A133-38 (to DHC, PI). Flow cytometry was supported, in part, by Massey Cancer Center Core NIH Grant P30 CA16059. Microscopy was performed at the VCU-Department of Anatomy and Neurobiology Microscopy Facility, supported, in part, by funding from NIH-NINDS Center Core Grant 5 P30 NS047463 and, in part, by funding from NIH-NCl Cancer Center Support Grant P30 CA016059. Macrophage MIF deficiency enhances immune response to Nippostrongylus brasiliensis.

\section{DISCLOSURE}

The authors declared no conflict of interest.

c 2017 Society for Mucosal Immunology

\section{REFERENCES}

1. World Health Organization. Preventive Chemotherapy in Human Helminthiasis: Coordinated Use of Antihelminthic Drugs in Control Interventions. (WHO Library Catalog Data, World Health Organization Press: Geneva, Switzerland, 2006).

2. Tchuem Tchuenté, L.A. Control of soil-transmitted helminths in subSaharan Africa: diagnosis, drug efficacy concerns and challenges. Acta Trop. 120, 4-11 (2011).

3. Wills-Karp, M. et al. Trefoil factor 2 rapidly induces interleukin 33 to promote type 2 immunity during allergic asthma and hookworm infection. J. Exp. Med. 209, 607-622 (2012).

4. Sutherland, T.E. et al. Chitinase-like proteins promote IL-17-mediated neutrophilia in a tradeoff between nematode killing and host damage. Nat. Immunol. 15, 1116-1125 (2014).

5. David, J.R. Delayed hypersensitivity in vitro: its mediation by cell-free substances formed by lymphoid cell-antigen interaction. Proc. Natl. Acad. Sci. USA 56, 72-77 (1966).

6. Bloom, B.R. \& Bennett, B. Mechanism of a reaction in vitro associated with delayed-type hypersensitivity. Science 153, 80-82 (1966).

7. Bernhagen, J. et al. MIF is a pituitary-derived cytokine that potentiates lethal endotoxaemia. Nature 365, 756-759 (1993).

8. Calandra, B.T., Bernhagen, J., Mitchell, R.A. \& Bucala, R. The macrophage is an important and previously unrecognized source of macrophage migration inhibitory factor. J. Exp. Med. 179, 1895-1902 (1994).

9. Calandra, T. et al. MIF as a glucocorticoid-induced modulator of cytokine production. Nature 377, 68-71 (1995).

10. Bacher, M. et al. An essential regulatory role for macrophage migration inhibitory factor in T-cell activation. Proc. Natl. Acad. Sci. USA 93, 7849-7854 (1996).

11. Makita, $\mathrm{H}$. et al. Effect of anti-macrophage migration inhibitory factor antibody on lipopolysaccharide-induced pulmonary neutrophil accumulation. Am. J. Respir. Crit. Care Med. 158, 573-579 (1998).

12. Rosengren, E. et al. The macrophage migration inhibitory factor MIF is a phenylpyruvate tautomerase. FEBS Lett. 417, 85-88 (1997).

13. Swope, M.D. \& Lolis, E. Macrophage migration inhibitory factor: cytokine, hormone, or enzyme? Rev. Physiol. Biochem. Pharmacol. 139, 1-32 (1999).

14. Rosengren, E. et al. The immunoregulatory mediator macrophage migration inhibitory factor (MIF) catalyzes a tautomerization reaction. Mol. Med. 2, 143-149 (1996).

15. Grieb, G., Merk, M., Bernhagen, J. \& Bucala, R. Macrophage migration inhibitory factor (MIF): a promising biomarker. Drug News Perspect. 23, 257-264 (2010).

16. Mitchell, R.A., Metz, C.N., Peng, T. \& Bucala, R. Sustained mitogenactivated protein kinase (MAPK) and cytoplasmic phospholipase A2 
activation by macrophage migration inhibitory factor (MIF). Regulatory role in cell proliferation and glucocorticoid action. J. Biol. Chem. 274, 1810018106 (1999).

17. Roger, T., David, J., Glauser, M.P. \& Calandra, T. MIF regulates innate immune responses through modulation of Toll-like receptor 4 . Nature 414, 920-924 (2001).

18. Kleemann, R. et al. Intracellular action of the cytokine MIF to modulate AP-1 activity and the cell cycle through Jab1. Nature 408, 211-216 (2000).

19. Chuang, C.-C. et al. Macrophage migration inhibitory factor regulates interleukin-6 production by facilitating nuclear factor-kappa B activation during Vibrio vulnificus infection. BMC Immunol. 11, 50 (2010).

20. Cross, J.V. et al. Nutrient isothiocyanates covalently modify and inhibit the inflammatory cytokine macrophage migration inhibitory factor (MIF). Biochem. J. 423, 315-321 (2009).

21. Lubetsky, J.B. et al. The tautomerase active site of macrophage migration inhibitory factor is a potential target for discovery of novel anti-inflammatory agents. J. Biol. Chem. 277, 24976-24982 (2002).

22. Mikulowska, A., Metz, C.N., Bucala, R. \& Holmdahl, R. Macrophage migration inhibitory factor is involved in the pathogenesis of collagen type llinduced arthritis in mice. J. Immunol. 158, 5514-5517 (1997).

23. Bernhagen, J. et al. MIF is a noncognate ligand of CXC chemokine receptors in inflammatory and atherogenic cell recruitment. Nat. Med 13, 587-596 (2007).

24. Bozza, M. et al. Targeted disruption of migration inhibitory factor gene reveals its critical role in sepsis. J. Exp. Med. 189, 341-346 (1999).

25. Koebernick, H. et al. Macrophage migration inhibitory factor (MIF) plays a pivotal role in immunity against Salmonella typhimurium. Proc. Natt. Acad. Sci. USA 99, 13681-13686 (2002).

26. Das, R. et al. Macrophage migration inhibitory factor (MIF) is a critical mediator of the innate immune response to Mycobacterium tuberculosis. Proc. Natl. Acad. Sci. USA 110, E2997-E3006 (2013).

27. Jüttner, S. et al. Migration inhibitory factor induces killing of Leishmania major by macrophages: dependence on reactive nitrogen intermediates and endogenous TNF-alpha. J. Immunol. 161, 2383-2390 (1998).

28. Satoskar, A.R., Bozza, M., Sosa, M.R., Lin, G. \& David, J.R. Migrationinhibitory factor gene-deficient mice are susceptible to cutaneous Leishmania major infection. Infect. Immun. 69, 906-911 (2001).

29. Rodríguez-sosa, M. et al. Macrophage migration inhibitory factor plays a critical role in mediating protection against the helminth parasite Taenia crassiceps. Infect. Immun. 71, 1247-1254 (2003).

30. Martiney, J.A. et al. Macrophage migration inhibitory factor release by macrophages after ingestion of Plasmodium chabaudi-infected erythrocytes: possible role in the pathogenesis of malarial anemia macrophage migration inhibitory factor release by macrophages after ingestion. Infect. Immun. 68, 2259-2267 (2000).

31. Chaisavaneeyakorn, S. et al. Immunity to placental malaria. IV. Placental malaria is associated with up-regulation of macrophage migration inhibitory factor in intervillous blood. J. Infect. Dis. 186, 1371-1375 (2002).

32. Rodriguez-Sosa, M., David, J.R., Bojalil, R., Satoskar, A.R. \& Terrazas, L.I. Cutting edge: susceptibility to the larval stage of the helminth parasite Taenia crassiceps is mediated by Th2 response induced via STAT6 signaling. J. Immunol. 168, 3135-3139 (2002).
33. Smith, K.A. \& Maizels, R.M. IL-6 controls susceptibility to helminth infection by impeding Th2 responsiveness and altering the Treg phenotype in vivo. Eur. J. Immunol. 44, 150-161 (2014).

34. Xiao, W. et al. Co-operative functions between nuclear factors NFkappaB and CCAT/enhancer-binding protein-beta (C/EBP-beta) regulate the IL-6 promoter in autocrine human prostate cancer cells. Prostate 61, 354-370 (2004).

35. Zhang, Y., Talalay, P., Cho, C.G. \& Posner, G.H. A major inducer of anticarcinogenic protective enzymes from broccoli: isolation and elucidation of structure. Proc. Natl. Acad. Sci. USA 89, 2399-2403 (1992).

36. Brown, K.K. et al. Direct modification of the proinflammatory cytokine macrophage migration inhibitory factor by dietary isothiocyanates. J. Biol. Chem. 284, 32425-32433 (2009).

37. Ouertatani-Sakouhi, H. et al. A new class of isothiocyanate-based irreversible inhibitors of macrophage migration inhibitory factor. Biochemistry 48, 9858-9870 (2009).

38. Calandra, T. \& Roger, T. Macrophage migration inhibitory factor: a regulator of innate immunity. Nat. Rev. Immunol. 3, 791-800 (2003).

39. Bancroft, A.J., Artis, D., Donaldson, D.D., Sypek, J.P. \& Grencis, R.K. Gastrointestinal nematode expulsion in IL-4 knockout mice is IL-13 dependent. Eur. J. Immunol. 30, 2083-2091 (2000).

40. Terrazas, L.I., Bojalil, R., Govezensky, T. \& Larralde, C. Shift from an early protective Th1-type immune response to a late permissive Th2-type response in murine cysticercosis (Taenia crassiceps). J. Parasitol. 84, 7481 (1998).

41. Stavitsky, A.B., Metz, C., Liu, S., Xianli, J. \& Bucala, R. Blockade of macrophage migration inhibitory factor (MIF) in Schistosoma japonicuminfected mice results in an increased adult worm burden and reduced fecundity. Parasite Immunol. 25, 369-374 (2003).

42. Simpson, K.D., Templeton, D.J. \& Cross, J.V. Macrophage migration inhibitory factor promotes tumor growth and metastasis by inducing myeloid-derived suppressor cells in the tumor microenvironment. $J$. Immunol. 189, 5533-5540 (2012).

43. Talalay, P., Dinkova-Kostova, A.T. \& Holtzclaw, W.D. Importance of phase 2 gene regulation in protection against electrophile and reactive oxygen toxicity and carcinogenesis. Adv. Enzyme Regul. 43, 121-134 (2003).

44. Healy, Z.R., Liu, H., Holtzclaw, W.D. \& Talalay, P. Inactivation of tautomerase activity of macrophage migration inhibitory factor by sulforaphane: a potential biomarker for anti-inflammatory intervention. Cancer Epidemiol. Biomarkers Prev. 20, 1516-1523 (2011).

45. Conine, S.J. \& Cross, J.V. MIF deficiency does not alter glucose homeostasis or adipose tissue inflammatory cell infiltrates during dietinduced obesity. Obesity 22, 418-425 (2014).

46. Camberis, M., Le Gros, G. \& Urban, J. Animal model of Nippostrongylus brasiliensis and Heligmosomoides polygyrus. Curr. Protoc. Immunol. 55:19.12: 19.12.1-19.12.27 (2003).

47. Caven, T.H. et al. IL-21 dependent IgE production in human and mouse in vitro culture systems is cell density and cell division dependent and is augmented by IL-10. Cell. Immunol. 238, 123-134 (2005).

48. Dussault, A.-A. \& Pouliot, M. Rapid and simple comparison of messenger RNA levels using real-time PCR. Biol. Proced. Online 8, 1-10 (2006).

49. Gommerman, J., Gommerman, J. \& Rojas, O. Creation of mixed bone marrow chimeras with appropriate controls. Protoc. Exch. (2011); doi:10.1038/protex.2011.269. 